EPJ Web of Conferences 71, 00004 (2014)

DOI: 10.1051/epjconf/20147100004

(C) Owned by the authors, published by EDP Sciences, 2014

\title{
Charm quarks as a probe of matter produced in relativistic nucleus-nucleus collisions
}

\author{
Yasir Ali a , Paweł Staszel \\ ${ }^{1}$ M. Smoluchowski Institute of Physics, Jagiellonian University, Reymonta 4, 30-059 Kraków Poland
}

\begin{abstract}
Direct measurement of hadrons containing charm quark carries important information about the initial stage of the nucleus-nucleus collision at relativistic energies. The study of open charm in $\mathrm{Pb}-\mathrm{Pb}$ collisions at SPS energies will be a powerful tool to investigate the production of heavy flavours and their interaction with the medium produced in such collisions. A feasibility study was initiated for the measurement of the $\mathrm{D}^{0}$ mesons (open charm) by its two-body decay into pion and kaon in central $\mathrm{Pb}-\mathrm{Pb}$ collision at SPS energies in NA61/SHINE experiment. To generate the physical input we used AMPT (A Multi Phase Transport Model) event generator and employed GEANT4 application to describe particle transport through the NA61/SHINE experimental setup supplemented by a future vertex detector (VD) that will allow for precise vertex reconstruction close to the primary interaction point. The results of the simulation shows that this measurement is feasible with a dedicated VD which allows the precise tracking close to the target.
\end{abstract}

\section{Introduction}

Direct measurement of hadrons containing charm quark carries important information about the initial stages of the nucleus-nucleus collision at relativistic energies. During the last 4 decades an approach based on perturbative Quantum Chromodynamics (pQCD) was formulated and used for the interpretation of open and hidden (charmonia) charm experimental data [1]. The approach is based on the assumption that $\mathrm{pQCD}$ can describe charm production in elementary hadronic and nuclear collisions and that charmonium creation is due to binding interaction between $c-\bar{c}$ quarks. To probe the hot and dense stages of the collision, one needs to measure observables that are particularly sensitive to the early moments of the collision, such as charmed particles, di-leptons and direct photons. Observables related to open charm mesons (i.e. particles containing one charm quark and one light quark) are expected to be particularly sensitive to the high density effects. The charm-quarks and hadrons are of vivid interest in the context of the phase transition between confined hadronic matter and the quark-gluon plasma (QGP) [2, 3].

In particular, the detection of $\mathrm{D}^{0}$ mesons in NA61/SHINE experiment is based on the reconstruction of their decay vertex and its distinction from the primary collision vertex. Due to the very low average proper decay length of the $\mathrm{D}^{0}$ meson (c $\tau$ is $123 \mu \mathrm{m}$ ) [4] the separation of the primary interaction vertex and the secondary decay vertex is difficult. This makes their detection particularly

ae-mail: yasir.ali@uj.edu.pl

This is an Open Access article distributed under the terms of the Creative Commons Attribution License 2.0, which permits unrestricted use, distribution, and reproduction in any medium, provided the original work is properly cited. 
challenging and imposes strong constraints on the detection system. The measurements of open charm in the NA61/SHINE experiment require good position resolution (on the level of few microns), low material budget, radiation tolerant and relatively fast vertex detector. In this work we have considered only the two body hadronic decay channel in which $\mathrm{D}^{0}$ mesons decays into pion and kaon with the branching ratio of $3.9 \%$ [5]. A feasibility study is developed for the measurement of the $\mathrm{D}^{0}$ mesons in $\mathrm{Pb}+\mathrm{Pb}$ collision at SPS energies. This study is done for the top SPS energy of $158 \mathrm{~A} \mathrm{GeV}$ and for the lower energy of $40 \mathrm{~A} \mathrm{GeV}$.

\section{Physics Motivation}

There is no direct measurement of open charm at SPS energies up to now. Charmonium $(J / \psi)$ has been already measured by NA38/NA50 and NA60 experiments but only at 158A GeV. Figure 1 depicts the measured $J / \psi$ production as a function of centrality expressed as the number of participating nucleons $\left(N_{\text {part }}\right)$, compared to $\mathrm{pQCD}$ predictions assuming normal nuclear absorption in the medium. The yields at the lower number of participants are consistent with pQCD, however there is a significant drop at $N_{\text {part }} \approx 200$ [7]. This effect is known as anomalous $J / \psi$ suppression and was often interpreted as a formation of QGP. The proposed maesurements will allow to verify whether similar anomalous effects are also present in open charm channel.

Recently, Satz [8] indicated that the simultaneous measurements of open charm and charmonia are needed to construct charm observables that are model independent. Such measurements would allow to disentangle between initial and final state effects revealing properties of hidden and open charm transport through the dense medium created in nucleus-nucleus collisions.

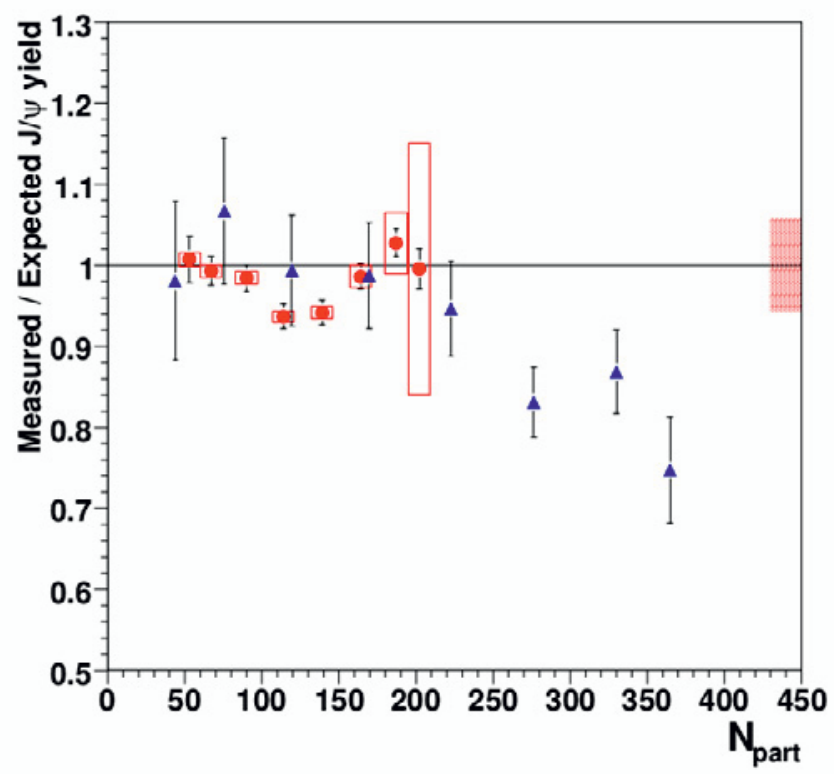

Figure 1. $J / \psi$ suppression pattern measured in $\mathrm{In}+\mathrm{In}$ and $\mathrm{Pb}+\mathrm{Pb}$ collisions, as a function of the number of participants $\mathrm{N}_{\text {part }}$ [7] The boxes around the $\mathrm{In}+\mathrm{In}$ points represnts correlated systematic errors. The filled box corresponds to the uncertainity in the absolute normalization of In+In points.

At RHIC low $p_{T}$ open charm production is provided by PHENIX and STAR collaborations $[9,10]$ and the relevant comparisons are depicted in Figure 2. 
As one of the examples Satz is discussing nuclear modification factors $\mathrm{R}_{A A}$ (which is most commonly used to study medium effects), measured at RHIC, at high and low transverse momentum $p_{T}$ for both $J / \psi$ and D mesons [9, 10]. The plot in Figure 2 (left side) depicts high transverse momenta at which $\mathrm{R}_{A A}$ for $J / \psi$ and $\mathrm{D}^{0}$ mesons are consistent. According to Satz such picture indicats no medium effects (no suppression nor enhancement) for $J / \psi$ as such effects would be seen as a deviation form open charm $\mathrm{R}_{A A}$. In contrary, at low $p_{T}$ (Figure 2 right side), $\mathrm{R}_{A A}(\mathrm{D})$ is consistent in unity, wheres $\mathrm{R}_{A A}$ of $(J / \psi)$ shows significant suppression, which increases with the centrality. This is a real effect of final state interaction. Such study performed for different energies and centralities should reveal a new phenomenology of charm dydamics in medium. It is important to compare hidded and open charm at the same phase space and provide preciese measurement of both charm states, e.g. measuremet of D mesons from there semi leptonic decays (as done so far at LHC and RHIC) should be replaces by direct measurement.
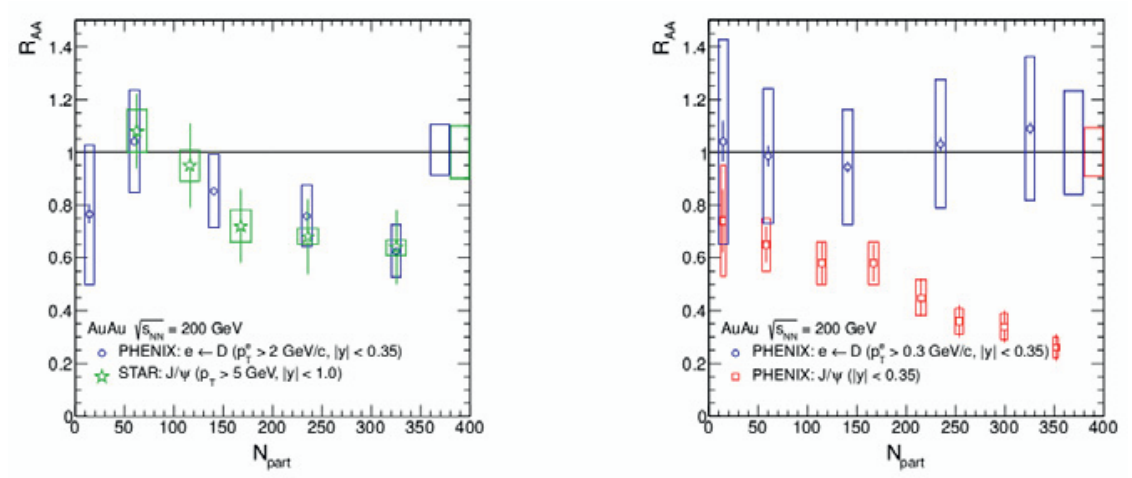

Figure 2. RHIC data from PHENIX and STAR $[9,10]$, comparing $J / \psi$ production to open charm production at high and low transverse momenta.

The NA60 collaboration published data on di-muon production in In+In collisions at the top SPS energy [11]. They confirmed the previously discovered di-muon enhancement [12] in the invariant mass range between $\phi$ mass and $J / \psi$ mass. Initially, this effect was connected to the open charm enhancement. The detailed analysis of spectra attributed to the enhanced component have shown a continues increase of the effective temperature with increasing invariant mass in the range below 1 $\mathrm{GeV} / \mathrm{c}^{2}$ followed by a relatively sudden drop (by $50 \mathrm{MeV}$ ) for masses above $1 \mathrm{GeV} / \mathrm{c}^{2}$. The observed increase of $\mathrm{T}_{\text {eff }}$ in the lower mass region can be attributed to hadronic sources coupled to the expanding medium, however, it was hard to interpret the drop above $1 \mathrm{GeV}$ to any known standard sources like Drell-Yan production or/and open charm fusion. The NA60 collaboration proposed the interpretation in terms of creation of the muon radiation source at the early collision phase that they attributed to QGP. This interpretation based strongly on the D mesons production limits deduced from the di-muon data in the model dependent way. The direct measurement of open charm production in nucleus-nucleus collisions at SPS energies will allow to verify the interpretation proposed by the NA60 collaboration.

\section{NA61/SHINE Experiment}

NA61/SHINE experiment is the fixed target experiment at CERN-SPS [13]. The setup includes the beam position detectors, Cherenkov counters and the scintillator stations located upstream of the 
target. These detectors provide information of timing, type and position of beam particles. For particle tracking the experiment uses two vertex time projection chambers (VTPC1 and VTPC2), gap TPC and two main TPCs. It also includes the time-of-flight detectors located behind main TPCs which are used for hadron identification, and the projectile spectator detector that measures the energy of projectile spectators delivering information on collision centrality. The future vertex detector will be located downstream of the target, at the distance of about $50 \mathrm{~cm}$ to the front of the active VTPC1 volume.

\section{Simulation and Results}

We performed a feasibility study for the $D^{0}$ meson measurement by its decay into two daughter particles, $D^{0} \rightarrow K^{-}+\pi^{+}$, in central $\mathrm{Pb}-\mathrm{Pb}$ collisions at the top SPS energy of $158 \mathrm{~A} \mathrm{GeV}$ and the lower energy of $40 \mathrm{~A} \mathrm{GeV}$. For a physical input we used AMPT event generator to generate 200k 0-10\% central events. AMPT predicts average multiplicity of 0.01 for $\mathrm{D}^{0}+\overline{D^{0}}$ per central $\mathrm{Pb}+\mathrm{Pb}$ event. This value seems to be underpredicted with respect to PYTHIA results scaled to number of binary nucleonnucleon collisions in central $\mathrm{Pb}+\mathrm{Pb}$ equal to 0.21 [14] as well as with respect to the prediction of the HSD model which is 0.2 . Because HSD model was tuned to properly describe available $\mathrm{p}+\mathrm{A}$ and $\pi+$ A charm production data at SPS energies [15], we scaled the AMPT multiplicities to the above prediction of the HSD model. Within the given statistics the AMPT event generator does not generate open charm at lower energy of $40 \mathrm{~A} \mathrm{GeV}$. However, we observe that the transverse mass slope parameter and the width of the rapidity distribution for kaons do not change by more than $10 \%$ going from $158 \mathrm{~A} \mathrm{GeV}$ to $40 \mathrm{~A} \mathrm{GeV}$. In order to describe phase space for $D^{0}$ at the lower energy, we assumed similar changes in shapes of distributions as that observed for kaons. The total yield was taken as predicted by the HSD model.

We employed GEANT4 to describe the particle transport through the NA61/SHINE experimental setup supplemented with the VD which allows for precise tracking at the target proximity. The VD modelled in GEANT4 consists of four detection stations VDS1, VDS2, VDS3 and VDS4 located respectively at distances of $5 \mathrm{~cm}, 10 \mathrm{~cm}, 15 \mathrm{~cm}$, and $20 \mathrm{~cm}$ downstream of the target. The track reconstruction is based on the hits generated during particle transport through vertex detector stations, VTPC1 and VTPC2. Only the tracks accepted in VTPC detectors are considered in the subsequent analysis. The acceptance is defined in the similar way as it is done in the standard NA61/SHINE analysis which is described in detail in [16]. We constructed the invariant mass distribution for kaon-pion pairs at the collision energy of $158 \mathrm{~A} \mathrm{GeV}$ and found, that the combinatorial background is few orders of magnitude higher than the $D^{0}+\overline{D^{0}}$ signal. In order to suppress the large combinatorial background, four cuts were incorporated, namely, the cut on the track transverse momentum $p_{T}$, the cut on the track impact parameter $d$, the cut on the longitudinal position $V_{z}$ of the track pair vertex relative to primary interaction point, and finaly, the cut on the parent particle impact parameter $D$. The first two are single-particle cuts, while the two other are two-particle cuts. It should be stressed that last three cuts require the precise tracking information at the target proximity delivered by the vertex detector..

The combinatorial invariant mass distributions after applying the whole set of background suppression cuts is depicted in Figure 3. The analysis assumes perfect particle identification (PID) is shown in Figure 3 (left). For perfect PID we obtain $\mathrm{S} / \mathrm{B} \approx 17$ and when we extrapolate these results to $50 \mathrm{M}$ central $\mathrm{Pb}+\mathrm{Pb}$ events (anticipated event number collected during 1 month run) the number of collected open charm mesons will be $(\sim 64000)$ with $S N R=246$. The Figure 3 (right) shows results obtained for analysis that does not use information on PID. In this case all the possible unlike sign pairs that survived the cuts have to be considered and for each pair two hypothesis are tested, namely that the first track in the pair is $K$ and the second one is $\pi$, and reversely, that the first track is $\pi$ and the second one in $K$. This procedure does not suppress the signal, however, the background increases 

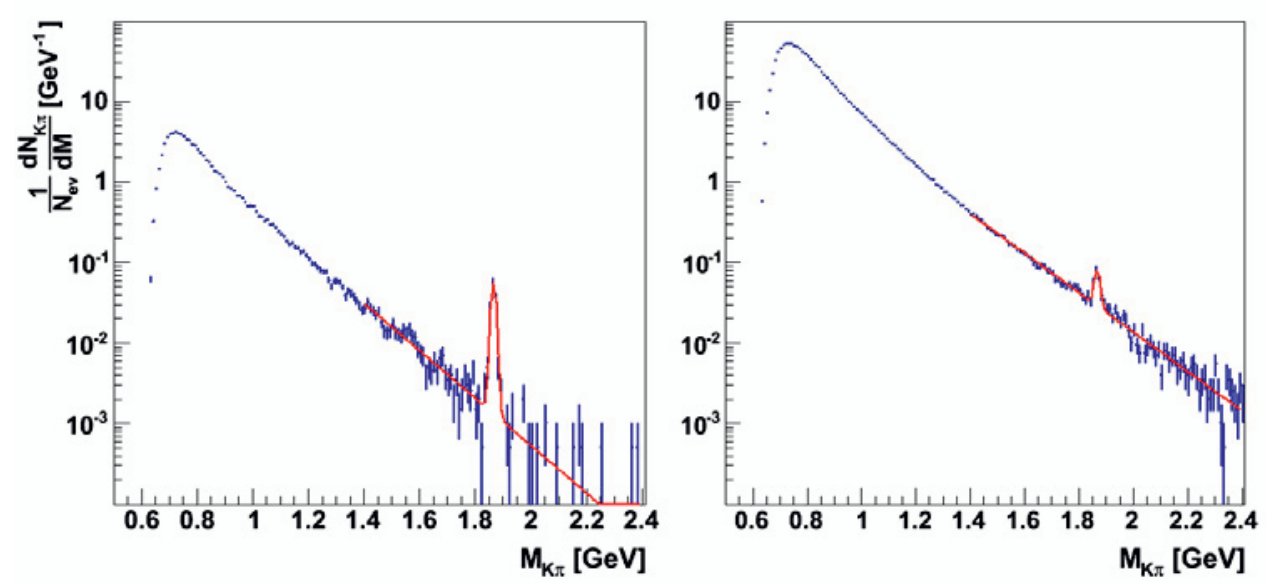

Figure 3. The invariant mass of pion-kaon pairs after full background reduction. The red curve represents fit with sum of exponential (background) and Gaussian function ( $D^{0}$ signal). Left: analysis done using PID information, right: analysis that does not utilize the PID information

noticeably which is seen in the figure. For the sample of $50 \mathrm{M}$ central $\mathrm{Pb}+\mathrm{Pb}$ events we will collect the same number of open charm mesons $(\sim 64000)$ as using perfect PID method, with $\mathrm{S} / \mathrm{B} \approx 1.0$ and $\mathrm{SNR}=197$. The simulation results also shows that even at the lower energy of $40 \mathrm{~A} \mathrm{GeV}$ there is significant yield of open charm mesons, namely, we will be able to collect about $2 \mathrm{~K}$ open charm mesons in $50 \mathrm{M}$ central $\mathrm{Pb}+\mathrm{Pb}$ events. The simulation results show, that the measurement of open charm in NA61/SHINE experiment is feasible if the current NA61/SHINE detector system is supplemented with a new vertex detector.

The mimimum ionizing MOS active pixel sensors (MIMOSA-26) [17] are selected as a detection element in the four VD stations. The chip is suitable for charge particles detection with the density of $10^{6}$ hits $/ \mathrm{cm}^{2} / \mathrm{s}$. The size of the chip is $13,7 \mathrm{~mm} \times 21,5 \mathrm{~mm}$ and the sensor matrix is composed by $576 \times 1152$ pixels of $18.4 \mu \mathrm{m}$ pitch. The pixel design is based on self biased diode architecture.

The design of the sensor is driven by the high readout frequency in order to keep the track multiplicity per frame at a low level. It is done by read out pixel columns in parallel, row by row. The chip readout time is $115.2 \mu \mathrm{s}$. Each pixel includes an amplification and Correlated Double Sampling (CDS) and each end of column is equipped with a discriminator [17]. The selection of MIMOSA-26 was mostly driven by the very high hit occupancy in the stations found from simulations. The hit occupancy in the VDS1 generated by a single central $\mathrm{Pb}+\mathrm{Pb}$ collision at $158 \mathrm{~A} \mathrm{GeV}$ reaches values of about 5 hits $/ \mathrm{mm}^{2}$ /event in the innermost part of the detector. Such a high occupancy cannot be handled by existing micro-pattern gas detectors mostly because of their one-dimensional readout. MIMOSA-26 offers, however, high pixel granularity with a real 2-dimensional readout. The sensor seems to fullfill all the requirements of the NA61/SHINE experiment in terms of radiation tolerance, time resolution, spatial resolution and material budget. The floor plan view of the MIMOSA-26 sensor is shown in Figure 4. 


\section{Block diagram of MIMOSA-26}

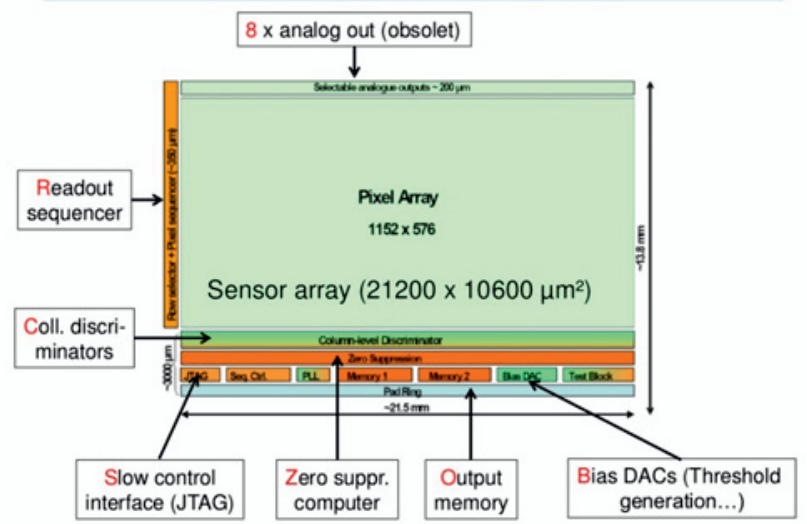

Figure 4. Schematic view of the MIMOSA-26 displaying the basic architecture of the chip.

\section{Conclusion}

Measurements of the heavy flavor mesons are important for better understanding of nucleus-nucleus reactions at relativistic energies. In this respect, there are experimental initiatives which measure charmonia states at SPS energies but so far there have been no direct open charm measurements at SPS energies. Our simulation results shows that the direct open charm measurement is feasible in the NA61/SHINE experiment by its upgradation with a dedicated vertex detector. The MIMOSA-26 chips seems feasible to use as a detection units for VD stations as it fullfill all the requirements imposed by the NA61/SHINE application and we forseen to use this device as a detection units for VD stations.

This contribution reports on the technical work motivated by the future experimental programme of the NA61/SHINE Collaboration at the CERN SPS. Authors gratefully acknowledge the contribution of NA61/SHINE to the results presented. This work is supported by the Foundation for Polish Science - MPD program, co-financed by the European Union within the European Regional Development Fund and the National Science Centre of Poland, grant UMO-2012/04/M/ST2/00816.

\section{References}

[1] M.Gazdzicki, C.Markert Acta. Phy. Pol. B. 4, Vol 31 (2000).

[2] U. Heinz, Nucl. Phys. A 661, 140c (1999).

[3] W. Cassing, E.L. Bratkovskaya, A. Sibirtsev, Nucl. Phys. A 691, 753 (2001).

[4] Amsler C et al. (Particle Data Group), PL B 667, 1 (2008).

[5] G. ORTONA. (ALICE collab.) Int. Journ. of Mod. Phys. Conf. Series. 2, 25-30, (2011).

[6] O.Linyk et al. International Journal of Modern Physics E (2008).

[7] E. Scomparina (NA60 collab. ) Recent results from the NA60 experiment (2009).

[8] H. Satz, arXiv:1303.3493v2 (2013).

[9] A. Adare et al. (PHENIX), Phys. Rev. C 84, 044905 (2011).

[10] A. Adare et al. (PHENIX), Phys. Rev. Lett. 98, 232301 (2007).

[11] R. Arnaldi et al.(NA60 Collab. ) arXiv:0810.3204v2 (2009). 


\section{ICNFP 2013}

[12] M. Masera (NA50 Collab. ), J. Phys. G: Part. Phys. 27, 667 (2001).

[13] The NA61/SHINE homepage [http://na61.web.cern.ch].

[14] P. Braun-Munzinger, J. Stachel, PLB 490196 (2000).

[15] W. Cassing, E.L Bratkovskaya, A. Sibirtsev, Nucl. Phys. A 691, 753 (2001).

[16] Y.Ali et al. Acta Phy. Pol. B. 10, Vol 44 (2013).

[17] MIMOSA26 User Manual, Institut Pluridisciplinaire Hubert Curien IN2P3-CNRS. 